\title{
Quantification of Biogenic Amines in 35 Korean Cottage Industry Traditional Gochujang (Fermented Red Pepper Paste) Products
}

\author{
Srinivasan Ramalingam ${ }^{1, \dagger}{ }^{\dagger}$ Ashutosh Bahuguna ${ }^{1, \dagger}$, SeMi Lim ${ }^{1, \dagger}$, Ah-ryeong Joe ${ }^{1}$, Jong-Suk Lee ${ }^{2}$, \\ So-Young Kim $^{3}$ (D) and Myunghee Kim $^{1, *}$
}

Citation: Ramalingam, S.; Bahuguna, A.; Lim, S.; Joe, A.-r.; Lee, J.-S.; Kim,

S.-Y.; Kim, M. Quantification of Biogenic Amines in 35 Korean Cottage Industry Traditional Gochujang (Fermented Red Pepper Paste) Products. Foods 2021, 10, 2370. https://doi.org/10.3390/ foods 10102370

Academic Editors: Maria Schirone and Pierina Visciano

Received: 4 August 2021

Accepted: 25 September 2021

Published: 6 October 2021

Publisher's Note: MDPI stays neutral with regard to jurisdictional claims in published maps and institutional affiliations.

Copyright: (c) 2021 by the authors. Licensee MDPI, Basel, Switzerland. This article is an open access article distributed under the terms and conditions of the Creative Commons Attribution (CC BY) license (https:// creativecommons.org/licenses/by/ $4.0 /)$.
1 Department of Food Science and Technology, Yeungnam University, Gyeongsan 38541, Korea; sribt27@gmail.com (S.R.); ashubahuguna@gmail.com (A.B.); thfvkalfpeh7@naver.com (S.L.); whdkfud12@naver.com (A.-r.J.)

2 Division of Food \& Nutrition and Cook, Taegu Science University, Daegu 41453, Korea; jslee1213@ynu.ac.kr

3 Department of Agrofood Resources, National Institute of Agricultural Sciences, Rural Development Administration, Wanju 55365, Korea; foodksy@korea.kr

* Correspondence: foodtech@ynu.ac.kr; Tel.: +82-53-810-2958

+ These authors contributed equally to this work.

Abstract: Traditional gochujang is well known for its distinguished flavor and taste. However the safety of cottage industry gochujang products is uncertain, particularly, in terms of biogenic amine (BA) content which is not yet documented. The present study aimed to determine the level of BAs present in 35 traditional gochujang products nationwide. All gochujang products had considerable amounts of total BAs ranging from $52.95 \mathrm{mg} / \mathrm{kg}$ to $176.24 \mathrm{mg} / \mathrm{kg}$. Individually, histamine and tyramine were either not detected or detected up to $16.94 \mathrm{mg} / \mathrm{kg}$ and $2.15-52.34 \mathrm{mg} / \mathrm{kg}$, respectively. In all the tested gochujang products, putrescine, spermidine, and spermine were detected in the range of $7.60-56.72 \mathrm{mg} / \mathrm{kg}, 14.96-36.93 \mathrm{mg} / \mathrm{kg}$, and $4.68-16.31 \mathrm{mg} / \mathrm{kg}$, respectively. A total of 22 and 19 gochujang products had less than $1 \mathrm{mg} / \mathrm{kg}$ of cadaverine and histamine, respectively. The findings indicate that all the gochujang products tested herein had BA levels below the suggested toxicity limits recommended by the various regulatory authorities, which reveal that they are safe for human consumption.

Keywords: fermentation; food safety; HPLC; histamine; principal component analysis; putrescine; red pepper paste

\section{Introduction}

Gochujang (fermented red pepper paste) is a traditional Korean fermented food that is generally used as a sauce in Korean cuisines and as a seasoning in spicy foods. In 2017, the total domestic retail market revenue of gochujang was 183.63 billion Korean won ( USD 149.55 million). Gochujang products were also exported to many countries, including the US, Japan, and China, generating a total of USD 31.98 million in revenue [1]. Gochujang is prepared as a paste by mixing various ingredients, such as powdered red pepper (Capsicum annuum L.), saccharified grain starch (rice, wheat, or barley), salt, powdered meju (natural soybean-based starter), and potable water. The mixture is then fermented and aged [2]. The primary ingredients of gochujang include red hot pepper powder, meju, and rice powder. In general, gochujang is prepared using two different methods: (1) traditional homemade method and (2) large-scale production in quality-controlled industries [3,4].

Household and cottage gochujang industries rely on traditional fermentation techniques using simple equipment, where fermentation is influenced by the natural microflora present in meju and surrounding environmental factors. However, large-scale industries employ controlled environment conditions and pure starter cultures, such as Aspergillus and Bacillus species, to produce gochujang [5]. The gochujang cottage industry in different 
provinces generates products with diversified nutritional values and organoleptic properties. Raw ingredients, process methods, microorganisms involved in the fermentation, and duration of the fermentation markedly influence the organoleptic properties of gochujang, including its aroma, taste, and texture [5]. Nowadays, consumers are highly interested in indigenous branded traditional gochujang products owing to the consistent outstanding sensory quality. However, the safety of gochujang products from the cottage industry is uncertain. These traditional cottage gochujang industries have minimal capital, and thus cannot afford to establish a quality control analysis department as part of their industry. The physicochemical, microbial, and toxicological profiles of these gochujang products are not available to the public to ensure consumer safety as they are not frequently monitored either by the production companies or by any food and health organization. Furthermore, the quality of gochujang products generated in the indigenous branded cottage industry has not been sufficiently examined [6].

BAs are organic nitrogenous compounds with low molecular weight that are mainly found in fermented foods. These are also microbial decarboxylation products of amino acids and are considered to be toxicants and the anti-nutritional elements of food [7]. An excessively high number of BAs, such as histamine ( $>500 \mathrm{ppm})$, putrescine $(>2000 \mathrm{ppm})$, spermine $(>600 \mathrm{ppm})$, and spermidine $(>600 \mathrm{ppm})$, induces considerable toxicity [7]. Notably, these nitrogenous compounds are mostly produced by microorganisms during fermentation through enzymatic decarboxylation of amino acids, as well as transamination of ketones and aldehydes [8]. Several studies have reported the presence of BAs in a wide range of food products, including fermented foods, different types of seafood, agricultural commodities [9], and baby foods, alcoholic beverages, and halal foods [10]. The number of BAs in different fermented foods varies and is associated with the type of available amino acids, the presence of BA-forming bacteria, and different factors that affect microbial activity, such as appropriate $\mathrm{pH}$, temperature, water activity, and time [10]. During the fermentation of gochujang, the native microbes convert the protein-rich raw materials (soybean in the meju) into different types of BAs, such as histamine, tyramine, $\beta$-phenylethylamine, tryptamine, putrescine, and cadaverine, as a result of the putrefactive process of proteins [11].

BAs are essential for cellular development and growth and are important regulators of several processes, such as brain activity, body temperature regulation, stomach $\mathrm{pH}$, gastric acid secretion, the immune response, and the synthesis of hormones and alkaloids [10]. However, the consumption of an excessive number of BAs leads to serious physiological and toxicological effects. Del Rio et al. [12] reported the harmful effect of BAs in boosting histamine toxicity, in addition to being responsible for the so-called "cheese reaction". BAs cause different levels of intoxication in different individuals owing to variations in genetic predisposition and changes in the level of mono and di-amine oxidase enzymes in the intestinal epithelium. Mono and di-amine oxidase enzymes are sensitive to BAs and impair the functioning of the small intestine or kidneys. Accordingly, celiac patients, people undergoing surgery, or those receiving treatment for cancer and other pathologies are affected by BAs [10]. The most frequent BA-related food intoxication is caused by histamine poisoning, previously known as scombroid fish poisoning as it was first reported to be caused by the Scombridae family, such as tuna. Secondary amines, such as putrescine and cadaverine, also play an important role in food poisoning, as they can increase the toxicity of histamine or react with nitrites to form carcinogenic nitrosamines [13]. Excessive consumption of BAs leads to serious implications, such as nausea, headache, heart and respiratory diseases, hypotension, and hypertension [14]. Therefore, the assessment of BAs is essential to ensure the safety of fermented foods.

Recently, studies on the anti-cholesterol, anti-obesity, and anti-atherosclerotic properties of gochujang have gained immense attention worldwide. Previously, several studies focused on improving the quality of gochujang by assessing the effects of additives and microflora on the organoleptic properties [15-17]. Additionally, numerous investigations have focused on the storage methods for extending the shelf life of gochujang, including physical 
sterilization methods and the addition of natural food ingredients [18]. Similarly, few studies have reported the BAs content of the large-scale industries and lab-made/home-made gochujang samples [19-21]. However, to the best of our knowledge, the quantity of BAs in gochujang products from the cottage industry has not been determined in any of the previous studies. Given the importance of BAs in the safety of fermented foods, it is essential to determine its content and evaluate other safety protocols of gochujang products to ensure consumer safety and inform consumer choice of appropriate gochujang products. Thus, the present study aimed to determine the quantity of various BAs, such as agmatine, cadaverine, histamine, 2-phenylethylamine, putrescine, spermidine, spermine, tryptamine, and tyramine, in 35 gochujang products collected from nationwide cottage industries.

\section{Materials and Methods}

\subsection{Chemicals}

Sodium hydroxide, sodium hydrogen carbonate, ammonium hydroxide, and perchloric acid were purchased from Junsei Chemicals (Seoul, Korea). Dansyl chloride and standard BAs were purchased from Sigma-Aldrich (St. Louis, MO, USA). High-performance liquid chromatography (HPLC)-grade ammonium acetate and acetonitrile were purchased from Merck (Damstadt, Germany). All chemicals used in this study were of analytical grade and were used as supplied.

\subsection{Sample Collection}

A total of 35 gochujang products were purchased from cottage industries in different provinces of the Republic of Korea. The major ingredients of gochujang products include red pepper powder, glutinous rice powder, powdered meju, grain syrup, malt, salt, and water. The codes and ingredients for the selected gochujang product are presented in Table 1.

\subsection{Estimation of $B A s$}

The BAs present in the gochujang were estimated using the protocol of Ramalingam et al. [22]. In brief, $5 \mathrm{~g}$ samples were extracted twice with $25 \mathrm{~mL}$ of $0.4 \mathrm{M}$ perchloric acid. Thereafter, the extracts were centrifuged at $4000 \times g$ for $10 \mathrm{~min}$ at $4{ }^{\circ} \mathrm{C}$, filtered using a Whatman paper No. 1, and subjected to dansyl chloride derivatization. The extracts were mixed with $2 \mathrm{M} \mathrm{NaOH}(200 \mu \mathrm{L})$, a saturated solution of $\mathrm{NaHCO}_{3}(300 \mu \mathrm{L})$, and $0.04 \mathrm{M}$ dansyl chloride $(2 \mathrm{~mL})$, and then incubated at $40{ }^{\circ} \mathrm{C}$ for $45 \mathrm{~min}$. After the incubation period, $100 \mu \mathrm{L}$ of $25 \% \mathrm{NH}_{4} \mathrm{OH}$ was added to the reaction mixture to stop the reaction. The volume of the mixture was increased to $5 \mathrm{~mL}$ using acetonitrile, to remove excess dansyl chloride. This mixture was then centrifuged $\left(2500 \times \mathrm{g}\right.$ for $5 \mathrm{~min}$ at $\left.4{ }^{\circ} \mathrm{C}\right)$ and eventually filtered through a $0.2 \mu \mathrm{m}$ membrane filter (Sartorius AG, Goettingen, Germany).

The BAs were quantified using an HPLC system (Thermo Fisher Scientific, Waltham, MA, USA) coupled with a UV-visible detector. A C18 column (5- $\mu \mathrm{m}$ pore size, $4.6 \times 250 \mathrm{~mm}$, XBridge Shield RP18, Waters Corporation, Milford, MA, USA) was used to separate the BAs by HPLC. In the HPLC program, the gradient flow rate of the mobile phase $(0.1 \mathrm{M}$ ammonium acetate and acetonitrile) was $1 \mathrm{~mL} / \mathrm{min}$ for $35 \mathrm{~min}$ and the column isothermal temperature was $30^{\circ} \mathrm{C}$. The sample $(20 \mu \mathrm{L})$ was injected by auto sample injection and analyzed at $254 \mathrm{~nm}$. Standard curves were plotted for known standard BAs, which were used for sample quantification.

\subsection{Statistical Analysis}

All experiments were carried out at least in triplicate, and the values are presented as mean \pm standard deviation. Statistical analyses were performed using SPSS software 23 (IBM, Chicago, IL, USA). One-way analysis of variance (ANOVA) in a completely randomized design and Duncan's multiple range comparison tests were used to determine significant differences between the samples with a 95\% confidence limit at $p<0.05$. The multivariate exploratory techniques of principal component analysis (PCA) were carried out to 
categorize the gochujang samples based on their quantities of BAs using Xlstat package on Microsoft Office Excel 2016 version.

Table 1. Details of the selected gochujang products.

\begin{tabular}{|c|c|c|}
\hline Product Code & $\begin{array}{l}\text { Location of the Company (in the } \\
\text { Republic of Korea) }\end{array}$ & Ingredients \\
\hline Go-1 & Jindo-gun, Jeollanam-do & garlic, meju* powder \\
\hline Go-2 & Chungju, Chungcheongbuk-do & soybean, apple extract \\
\hline Go-3 & Cheongyang-gun, Chungcheongnam-do & red pepper powder, sea salt, maesil ** \\
\hline Go-4 & Jeongeup-si, Jeollabuk-do & red pepper powder, sea salt, meju powder \\
\hline Go-5 & Yesan-gun, Chungcheongnam-do & $\begin{array}{l}\text { jujube cherry tomato puree, apple enzyme, grain syrup, } \\
\text { red pepper powder, sea salt, yeast }\end{array}$ \\
\hline Go-6 & Yesan-gun, Chungcheongnam-do & $\begin{array}{c}\text { rice grain syrup, apple enzyme, red pepper powder, meju, } \\
\text { salt, yeast }\end{array}$ \\
\hline Go-7 & Eumseong-gun, Chungcheongbuk-do & red pepper powder, shiitake mushroom, soybean, salt \\
\hline Go-8 & Daegu-si & red pepper powder, maesil sugar syrup, garlic, salt \\
\hline Go-9 & Sunchang-gun, Jeollabuk-do & red pepper powder, sea salt, glutinous rice, malt \\
\hline Go-10 & Sunchang-gun, Jeollabuk-do & red pepper powder, sea salt, barley, malt \\
\hline Go-11 & Sunchang-gun, Jeollabuk-do & red pepper powder, sea salt, maesil, malt \\
\hline Go-12 & Sunchang-eup, Jeollabuk-do & glutinous rice, meju powder, rice flour, malt, sea salt \\
\hline Go-13 & Sunchang-eup, Jeollabuk-do & glutinous rice, sea salt, malt, maesil sugar syrup \\
\hline Go-14 & Sunchang-eup, Jeollabuk-do & $\begin{array}{c}\text { red pepper powder, glutinous rice, meju powder, rice flour, malt, } \\
\text { sea salt }\end{array}$ \\
\hline Go-15 & Sunchang-gun, Jeollabuk-do & $\begin{array}{l}\text { red pepper powder, glutinous rice, sea salt, malt, meju powder, } \\
\text { maesil sugar syrup }\end{array}$ \\
\hline Go-16 & Sunchang-gun, Jeollabuk-do & red pepper powder, glutinous rice, sea salt, malt, mеju powder \\
\hline Go-17 & Sunchang-gun, Jeollabuk-do & red pepper powder, barley, sea salt, malt, mејu powder \\
\hline Go-18 & Iksan-si, Jeollabuk-do & $\begin{array}{l}\text { germinated brown rice, red pepper powder, malt, } \\
\text { rice grain syrup }\end{array}$ \\
\hline Go-19 & Gangjin-gun, Jeollanam-do & red pepper powder, loofah enzyme, sea salt \\
\hline Go-20 & Hamyang-gun, Gyeongsangnam-do & $\begin{array}{c}\text { red pepper powder, malt, rice grain syrup, glutinous rice, } \\
\text { bamboo salt, alcohol }\end{array}$ \\
\hline Go-21 & Hamyang-gun, Gyeongsangnam-do & glutinous rice, malt, red pepper powder, bamboo salt \\
\hline Go-22 & Seocheon-gun, Chungcheongnam-do & soybean, sea salt, glutinous rice flour, red chili powder \\
\hline Go-23 & Seoul & traditional meju, glutinous rice flour, malt \\
\hline Go-24 & Sunchang-gun, Jeollabuk-do & $\begin{array}{c}\text { red pepper powder, glutinous rice, meju powder, rice flour, malt, } \\
\text { sea salt }\end{array}$ \\
\hline Go-25 & Hamyang-gun, Gyeongsangnam-do & red pepper powder, glutinous rice, malt, meju powder, sea salt \\
\hline Go-26 & Yecheon-gun, Gyeongsangbuk-do & red pepper powder, mеju powder, sea salt \\
\hline Go-27 & Iksan-si, Jeollabuk-do & $\begin{array}{l}\text { sancho }{ }^{* * *} \text { pepper powder, meju powder, sea salt, barley powder, } \\
\text { maesil sugar syrup, glutinous rice powder }\end{array}$ \\
\hline Go-28 & Bucheon-si, Gyeonggi-do & $\begin{array}{c}\text { red pepper powder, meju powder, cheonggukjang }{ }^{* * * *} \text { powder, } \\
\text { barley, malt }\end{array}$ \\
\hline Go-29 & Haenam-gun, Jeollanam-do & $\begin{array}{l}\text { red pepper powder, glutinous rice, rice grain syrup, cider, } \\
\text { shoch }^{* * * *}, \text { meju powder, sugar }\end{array}$ \\
\hline Go-30 & Pocheon-si, Gyeonggi-do & $\begin{array}{l}\text { red pepper powder, glutinous rice, rice grain syrup, malt, } \\
\text { meju powder, sea salt }\end{array}$ \\
\hline Go-31 & Bonghwa-gun, Gyeongsangbuk-do & red pepper powder, glutinous rice, mејu powder, malt, sea salt \\
\hline Go-32 & Pyeongchang-gun, Gangwon-do & $\begin{array}{l}\text { red pepper powder, meju powder, glutinous rice powder, } \\
\text { maesil sugar syrup, malt, rice grain syrup }\end{array}$ \\
\hline Go-33 & Gunsan-si, Jeollabuk-do & red pepper powder, rice, sea salt, meju powder, malt \\
\hline Go-34 & Jangheung-gun, Jeollanam-do & red pepper powder, sea salt \\
\hline Go-35 & Gongju-si, Chungchungnam-do & red pepper powder, glutinous rice, sea salt, soybean, malt \\
\hline
\end{tabular}

$M e j u^{*}$ : Brick of fermented soybean produced by pounding, kneading, and shaping the cooked soybeans followed by a long period of fermentation in the natural or conditioned environment; Maesil **: Prunus mume Siebold and Zucc; Sancho ***: Zanthoxylum piperitum (L.) DC.; Cheonggukjang ****: Fast fermented soybean paste, produced by fermenting the boiled soybeans with Bacillus subtilis for 2-4 days; Shoch $u^{* * * * *}$ : A Korean alcoholic beverage containing $16.9-19 \%$ ethanol. 


\section{Results and Discussion}

Gochujang fabricated in the traditional cottage industry contains a mixture of complex raw materials (including $m e j u$, a protein rich substrate), which are fermented by different microorganisms and are thus more vulnerable to BA production [1]. Histamine, tyramine, cadaverine, 2-phenylethylamine, spermine, spermidine, putrescine, tryptamine, and agmatine are the predominant BAs present in fermented foods [7]. Typically, excess BAs in foods are produced by the action of microorganisms. Therefore, the level of BAs in foods is an indirect measure of the microbial load [7]. As BAs are one of the main toxicants in fermented foods, we assessed their presence in 35 gochujang products from different cottage industries found nationwide and their impact on the quality and safety of the products.

The total BAs in all gochujang samples ranged from $52.95 \mathrm{mg} / \mathrm{kg}$ to $176.24 \mathrm{mg} / \mathrm{kg}$. The highest amount of BA was observed in Go-8 $(176.24 \pm 3.24 \mathrm{mg} / \mathrm{kg})$, followed by Go-22 (142.11 $\pm 1.12 \mathrm{mg} / \mathrm{kg})$, Go-28 (105.05 $\pm 0.36 \mathrm{mg} / \mathrm{kg})$, Go-11 (106.76 $\pm 1.85 \mathrm{mg} / \mathrm{kg})$, and Go-33 $(100.03 \pm 5.55 \mathrm{mg} / \mathrm{kg})$, whereas the least amount was detected in Go-20 $(52.95 \pm 0.32 \mathrm{mg} / \mathrm{kg}$ ) (Figure 1). Based on the total BAs, the gochujang samples were further classified into three categories: group I, not detected level to $75 \mathrm{mg} / \mathrm{kg}$ BAs; group II, 75-100 mg/kg BAs; and group III, greater than $100 \mathrm{mg} / \mathrm{kg}$ BAs. The majority of gochujang samples (45.71\%) fell in group I, followed by group II (40\%) and group III (14.28\%). Although there are no strict guidelines for total BAs in fermented food, many reports suggest $1000 \mathrm{mg} / \mathrm{kg}$ as the maximum permissible limit [23]. The variation in BA content in the different gochujang products is due to several reasons; however, the most evident is the different raw materials used to prepare the products. Microbial population and physiochemical conditions also significantly contributed to the variations. The gochujang samples tested herein had BAs within the suggested limit $(<1000 \mathrm{mg} / \mathrm{kg})$, and the results were consistent with those of published reports, demonstrating a similar type of BA profile in various gochujang samples [19-21]. The low amount of the BAs in the gochujang samples might be due to excellent hygienic conditions, selection of raw materials, and fermentation environments, which are the major factors that influence the production of BAs.

Among the different BAs, histamine has garnered more attention due to its severe toxic effect (scombroid poisoning) [7]. Therefore, most regulatory bodies, such as the US Food and Drug Administration (US FDA) and the European Food Safety Authority, emphasize the histamine level in foods. According to the US FDA guidelines, $50 \mathrm{mg} / \mathrm{kg}$ histamine is the legal limit for fishes [24], whereas the European Commission has set $100 \mathrm{mg} / \mathrm{kg}$ as the permissible limit in fishes and $400 \mathrm{mg} / \mathrm{kg}$ in fish sauces produced by fermentation [25]. The World Health Organization also suggested $200 \mathrm{mg} / \mathrm{kg}$ as the maximum permissible limit of histamine in food products [26]. Similarly, the regulatory authorities of Korea and China suggested $200-400 \mathrm{mg} / \mathrm{kg}$ of histamine as the maximum permissible limit in food products $[27,28]$. In the 35 gochujang products tested herein, histamine levels ranged from not detectable to $16.94 \mathrm{mg} / \mathrm{kg}$ (Figure 2 and Table 2). The maximum amount of histamine was detected in Go-28 $(16.94 \pm 0.03 \mathrm{mg} / \mathrm{kg})$, whereas the least amount was detected in Go-25, Go-27, and Go-32. Based on the histamine level, the samples were divided into three categories: group I, no detection level to $5 \mathrm{mg} / \mathrm{kg}$ histamine; group II, 5-10 mg/kg histamine; and group III, >10 mg/ kg histamine. Most of the gochujang samples $(82.86 \%)$ were assigned to group I, followed by group III (11.43\%) and group II (5.71\%). The gochujang samples Go-1 (10.36 $\pm 0.17 \mathrm{mg} / \mathrm{kg})$, Go-11 (11.27 $\pm 0.02 \mathrm{mg} / \mathrm{kg})$, Go$28(16.94 \pm 0.03 \mathrm{mg} / \mathrm{kg})$, and Go-33 $(15.54 \pm 2.16 \mathrm{mg} / \mathrm{kg})$ had histamine levels $>10 \mathrm{mg} / \mathrm{kg}$. Nonetheless, the histamine level in all samples was below the suggested toxicity limit recommended by various regulatory authorities, suggesting that the 35 gochujang products were safe for consumption based on their histamine levels. The results align well with those of Lee et al. [21] and Cho et al. [19], thereby highlighting a similar type of histamine level in different gochujang samples. However, Kim et al. [20] reported slightly higher histamine levels $(2.2-59.0 \mathrm{mg} / \mathrm{kg})$ in eight gochujang samples. 


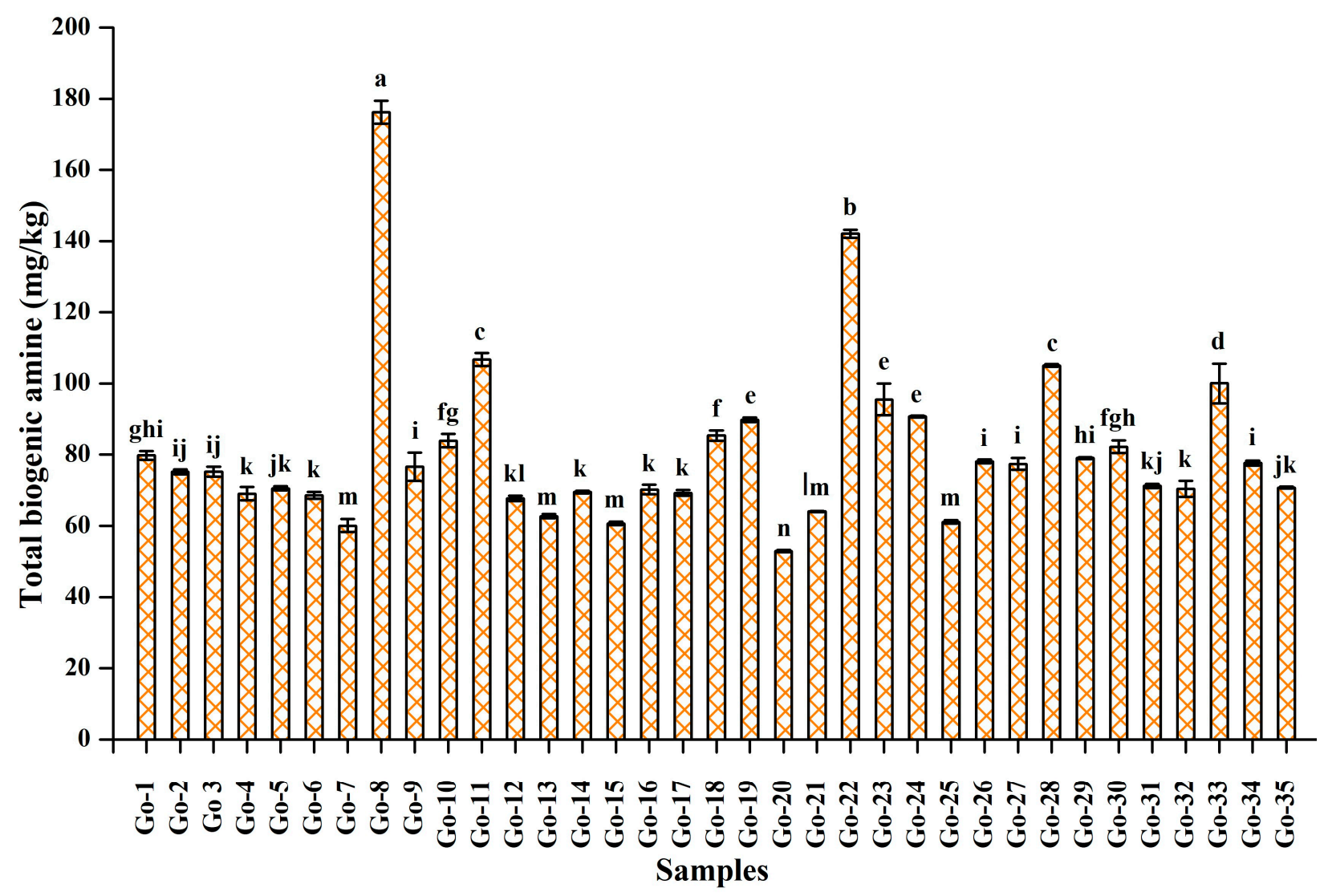

Figure 1. Total biogenic amines quantity in 35 gochujang products from the cottage industry. Different lowercase letters on the bars indicate significant differences $(p<0.05)$ based on Duncan's multiple comparison test.

Tyramine is a concerning BA in food, is responsible for the cheese reaction, and causes syndromes, such as histamine poisoning [29]. The regulatory authorities have not provided strict guidelines for tyramine. However, few reports suggest that the toxic limit of tyramine is $100-800 \mathrm{mg} / \mathrm{kg}[23,30]$. Herein, tyramine was detected to range from $2.15-52.34 \mathrm{mg} / \mathrm{kg}$ in the tested gochujang samples (Figure 2 and Table 2); these levels are below the recommended toxicity limit suggested by the regulatory authorities [23,30]. The highest amount of tyramine was detected in Go-8 $(52.34 \pm 0.41 \mathrm{mg} / \mathrm{kg})$, whereas the least amount was in Go-7 $(2.15 \pm 0.03 \mathrm{mg} / \mathrm{kg})$. Based on the level of tyramine, we further divided the gochujang samples into three groups: group I, no detection level to $5 \mathrm{mg} / \mathrm{kg}$ tyramine; group II, $5-20 \mathrm{mg} / \mathrm{kg}$ tyramine; and group III, $>20 \mathrm{mg} / \mathrm{kg}$ tyramine. Most gochujang samples were assigned to group I (65.71\%), followed by group II (28.57\%) and group III (5.71\%). The level of tyramine in the samples was below the toxicity level of $100-800 \mathrm{mg} / \mathrm{kg}[23,30]$ and aligned with the levels found by Kim et al. [20] in 8 gochujang samples (range of $2.9-126.8 \mathrm{mg} / \mathrm{kg}$ ).

Agmatine is another concerning BA in foods that is formed by the decarboxylation of L-arginine and is readily converted into putrescine [31,32]. In the gochujang samples, agmatine was either not detected or detected up to $29.78 \mathrm{mg} / \mathrm{kg}$, with the highest amount found in Go-22 (29.78 $\pm 0.12 \mathrm{mg} / \mathrm{kg})$ (Figure 2 and Table 2). Based on the detected amount of agmatine, the samples were divided into three groups: group I, not detected level to $10 \mathrm{mg} / \mathrm{kg}$ agmatine; group II, $10-20 \mathrm{mg} / \mathrm{kg}$ agmatine; and group III, $>20 \mathrm{mg} / \mathrm{kg}$ agmatine. Most of the gochujang samples were assigned to group II (68.57\%), followed by group III $(17.14 \%)$ and group I (14.28\%).

Putrescine, spermidine, and spermine are common BAs found in foods. The active role of spermidine and spermine as food allergens has been reported by several researchers [33-35]. To date, no legal limit has been recommended for putrescine, spermidine, and spermine; however, many studies suggest oral toxicity limits of $2000 \mathrm{mg} / \mathrm{kg}$, $600 \mathrm{mg} / \mathrm{kg}$, 
and $600 \mathrm{mg} / \mathrm{kg}$, respectively [7,36]. In the 35 gochujang samples assessed herein, putrescine, spermidine, and spermine were detected in the range of $7.60-56.72 \mathrm{mg} / \mathrm{kg}$, $14.96-36.93 \mathrm{mg} / \mathrm{kg}$, and $4.68-16.31 \mathrm{mg} / \mathrm{kg}$, respectively (Figure 2 and Table 2). The maximum amount of putrescin was detected in Go-8 $(56.72 \pm 0.50 \mathrm{mg} / \mathrm{kg})$, whereas the least amount was detected in Go-21 $(7.60 \pm 0.01 \mathrm{mg} / \mathrm{kg})$. The maximum amount of spermidine $(36.93 \pm 0.20 \mathrm{mg} / \mathrm{kg})$ and spermine $(16.31 \pm 0.04 \mathrm{mg} / \mathrm{kg})$ was detected in Go-19 and Go-5, respectively, whereas the least amount of spermidine $(14.96 \pm 0.02 \mathrm{mg} / \mathrm{kg})$ and spermine $(4.68 \pm 0.01 \mathrm{mg} / \mathrm{kg})$ was detected in Go-20. These findings are consistent with the reports that demonstrated variations in these BAs in different gochujang samples [19,20]. Lee et al. [21] determined the presence of BAs in eight gochujang products and found that the amount of putrescine and spermidine ranged $0.3-2.6 \mathrm{mg} / \mathrm{kg}$ and $0.6-1.3 \mathrm{mg} / \mathrm{kg}$, respectively. Unlike our results, Lee et al. [21] detected a lower amount of putrescine and spermidine in the gochujang samples tested. This variation might be due to different raw materials used and the involvement of microbial population during the fermentation of gochujang.

2-Phenylethylamine is one of the most frequently found BAs in foods and together with other BAs, provokes food-induced migraine and hypertension [37]. There is no legal limit for 2-phenylethylamine in food samples; however, few independent researchers suggested $30 \mathrm{mg} / \mathrm{kg}$ as the toxic threshold [30]. Herein, 2-phenylethylamine was either not detected or detected up to $26.23 \mathrm{mg} / \mathrm{kg}$ in the gochujang samples, with the highest amount found in Go-22 (26.23 $\pm 0.08 \mathrm{mg} / \mathrm{kg}$; Figure 2 and Table 2). These results align well with those of previous studies [19-21], thereby indicating that the content of 2-phenylethylamine varies in different gochujang samples. Kim et al. [20] tested gochujang samples and found $1.8-24.8 \mathrm{mg} / \mathrm{kg}$ of 2-phenylethylamine, which is consistent with the present findings. Compared with the present study, Lee et al. [21] found a lower amount of 2-phenylethylamine (range of $0.5-2.0 \mathrm{mg} / \mathrm{kg}$ ).

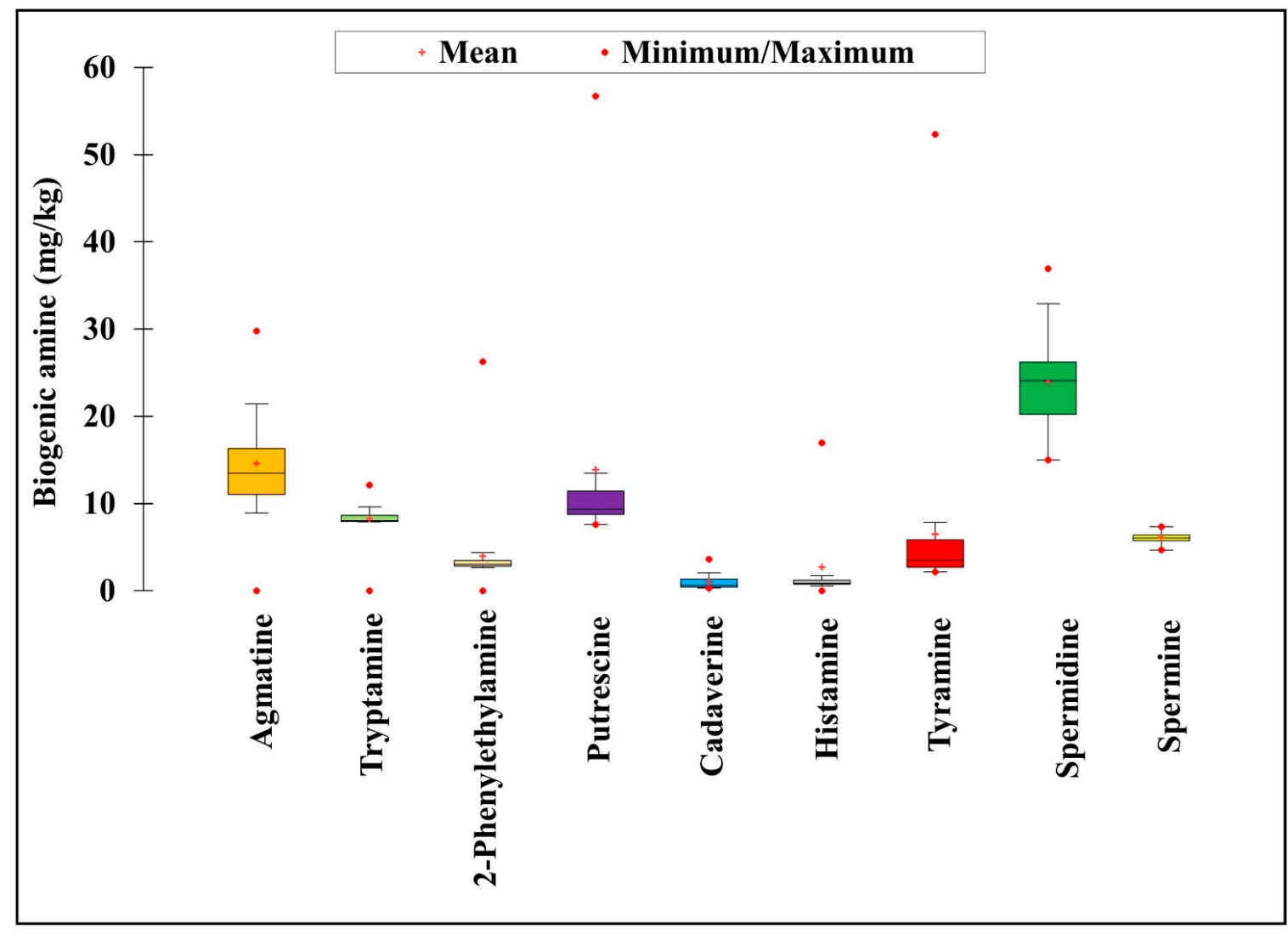

Figure 2. Mean quantity of various biogenic amines in the 35 traditional cottage industry gochujang products. 
Table 2. Quantity of different biogenic amines $(\mathrm{mg} / \mathrm{kg})$ in the 35 gochujang products from the traditional cottage industry.

\begin{tabular}{|c|c|c|c|c|c|c|c|c|c|}
\hline Samples & Agmatine & Tryptamine & 2-Phenylethylamine & Putrescine & Cadaverine & Histamine & Tyramine & Spermidine & Spermine \\
\hline Go-1 & $8.92 \pm 0.34$ & $7.95 \pm 0.00$ & $2.67 \pm 0.00$ & $10.13 \pm 0.18$ & $1.42 \pm 0.11$ & $10.36 \pm 0.17$ & $3.69 \pm 0.11$ & $27.47 \pm 0.17$ & $7.19 \pm 0.03$ \\
\hline Go-2 & $8.89 \pm 0.14$ & $8.51 \pm 0.02$ & $2.90 \pm 0.02$ & $9.78 \pm 0.03$ & $0.75 \pm 0.04$ & $0.77 \pm 0.04$ & $6.91 \pm 0.09$ & $30.37 \pm 0.36$ & $6.32 \pm 0.01$ \\
\hline Go 3 & $10.60 \pm 0.10$ & $10.35 \pm 0.16$ & $2.70 \pm 0.01$ & $10.25 \pm 0.29$ & $0.68 \pm 0.01$ & $0.60 \pm 0.20$ & $5.56 \pm 0.15$ & $28.62 \pm 0.58$ & $5.88 \pm 0.07$ \\
\hline Go-5 & $9.99 \pm 0.19$ & $8.10 \pm 0.01$ & $2.73 \pm 0.01$ & $10.06 \pm 0.06$ & $0.35 \pm 0.01$ & $1.71 \pm 0.02$ & $5.36 \pm 0.10$ & $25.90 \pm 0.16$ & $16.31 \pm 0.04$ \\
\hline Go-6 & $12.25 \pm 0.36$ & $7.97 \pm 0.01$ & $2.71 \pm 0.02$ & $8.30 \pm 0.02$ & $0.39 \pm 0.00$ & $0.77 \pm 0.10$ & $4.67 \pm 0.04$ & $26.69 \pm 0.36$ & $5.76 \pm 0.46$ \\
\hline Go-7 & $9.24 \pm 0.06$ & $7.90 \pm 0.01$ & $3.36 \pm 0.09$ & $8.61 \pm 0.07$ & $0.27 \pm 0.01$ & $0.54 \pm 0.00$ & $2.15 \pm 0.03$ & $22.48 \pm 1.40$ & $5.47 \pm 0.12$ \\
\hline Go-8 & $19.60 \pm 0.90$ & $8.90 \pm 0.02$ & $4.35 \pm 0.08$ & $56.72 \pm 0.50$ & $0.99 \pm 0.03$ & $0.81 \pm 0.00$ & $52.34 \pm 0.41$ & $24.16 \pm 1.29$ & $6.39 \pm 0.01$ \\
\hline Go-10 & $15.86 \pm 0.92$ & $7.97 \pm 0.00$ & $3.08 \pm 0.00$ & $10.94 \pm 0.35$ & $0.57 \pm 0.01$ & $5.47 \pm 0.30$ & $7.82 \pm 0.02$ & $26.36 \pm 0.10$ & $5.81 \pm 0.00$ \\
\hline Go-11 & $10.66 \pm 1.12$ & $7.88 \pm 0.00$ & $4.46 \pm 0.03$ & $24.24 \pm 0.10$ & $3.20 \pm 0.16$ & $11.27 \pm 0.02$ & $17.05 \pm 0.30$ & $22.36 \pm 0.09$ & $5.59 \pm 0.01$ \\
\hline Go-12 & $13.13 \pm 0.41$ & $7.92 \pm 0.01$ & $2.72 \pm 0.00$ & $9.07 \pm 0.03$ & $0.38 \pm 0.12$ & $1.18 \pm 0.02$ & $2.75 \pm 0.02$ & $24.57 \pm 0.24$ & $6.00 \pm 0.01$ \\
\hline Go-13 & $11.87 \pm 0.40$ & $7.91 \pm 0.10$ & $2.98 \pm 0.01$ & $8.50 \pm 0.02$ & $0.39 \pm 0.00$ & $1.19 \pm 0.07$ & $2.94 \pm 0.07$ & $21.35 \pm 0.03$ & $5.53 \pm 0.01$ \\
\hline Go-14 & $15.58 \pm 0.22$ & $7.89 \pm 0.00$ & $2.83 \pm 0.00$ & $8.69 \pm 0.00$ & $1.37 \pm 0.01$ & $0.86 \pm 0.00$ & $3.02 \pm 0.02$ & $23.48 \pm 0.05$ & $5.75 \pm 0.04$ \\
\hline Go-15 & $11.35 \pm 0.37$ & $7.90 \pm 0.00$ & $2.74 \pm 0.00$ & $8.92 \pm 0.02$ & $0.41 \pm 0.00$ & $0.76 \pm 0.01$ & $2.70 \pm 0.01$ & $2.70 \pm 0.03$ & $5.58 \pm 0.02$ \\
\hline Go-16 & $15.61 \pm 1.24$ & $7.90 \pm 0.00$ & $2.93 \pm 0.00$ & $9.22 \pm 0.02$ & $1.44 \pm 0.01$ & $0.77 \pm 0.03$ & $3.19 \pm 0.00$ & $23.28 \pm 0.02$ & $5.82 \pm 0.01$ \\
\hline Go-17 & $15.08 \pm 0.25$ & $7.92 \pm 0.00$ & $2.81 \pm 0.00$ & $7.63 \pm 0.00$ & $0.41 \pm 0.01$ & $1.06 \pm 0.01$ & $2.59 \pm 0.06$ & $25.33 \pm 0.17$ & $6.37 \pm 0.32$ \\
\hline Go-18 & $25.54 \pm 0.10$ & $8.04 \pm 0.01$ & $3.16 \pm 0.02$ & $11.23 \pm 0.07$ & $2.03 \pm 0.00$ & $0.72 \pm 0.02$ & $7.81 \pm 0.15$ & $19.87 \pm 0.67$ & $6.94 \pm 0.08$ \\
\hline Go-20 & $11.44 \pm 0.23$ & $7.89 \pm 0.01$ & $2.92 \pm 0.01$ & $7.72 \pm 0.01$ & $0.31 \pm 0.01$ & $0.72 \pm 0.03$ & $2.28 \pm 0.00$ & $14.96 \pm 0.02$ & $4.68 \pm 0.01$ \\
\hline Go-21 & $15.52 \pm 0.03$ & $8.04 \pm 0.02$ & $2.84 \pm 0.00$ & $7.60 \pm 0.01$ & $0.31 \pm 0.01$ & $1.16 \pm 0.01$ & $2.28 \pm 0.00$ & $20.18 \pm 0.09$ & $6.05 \pm 0.01$ \\
\hline Go-22 & $29.78 \pm 0.12$ & $8.04 \pm 0.00$ & $26.23 \pm 0.08$ & $31.42 \pm 0.04$ & $1.58 \pm 0.02$ & $0.82 \pm 0.01$ & $14.90 \pm 0.16$ & $22.46 \pm 0.55$ & $6.85 \pm 0.14$ \\
\hline Go-23 & $\mathrm{ND}^{*}$ & $8.06 \pm 0.07$ & $8.47 \pm 0.71$ & $41.61 \pm 4.42$ & $1.16 \pm 0.06$ & $0.72 \pm 0.06$ & $6.05 \pm 0.48$ & $20.28 \pm 0.36$ & $6.61 \pm 0.08$ \\
\hline Go-24 & $21.16 \pm 0.05$ & $12.11 \pm 0.05$ & $2.85 \pm 0.02$ & $13.30 \pm 0.06$ & $0.84 \pm 0.03$ & $1.20 \pm 0.02$ & $5.23 \pm 0.01$ & $26.80 \pm 0.01$ & $7.24 \pm 0.02$ \\
\hline Go-25 & $12.25 \pm 0.34$ & $7.89 \pm 0.00$ & $3.43 \pm 0.02$ & $9.25 \pm 0.03$ & $0.55 \pm 0.08$ & ND * & $2.43 \pm 0.00$ & $19.76 \pm 0.02$ & $5.55 \pm 0.01$ \\
\hline Go-26 & $21.02 \pm 0.13$ & $7.96 \pm 0.00$ & $3.03 \pm 0.00$ & $9.37 \pm 0.01$ & $0.38 \pm 0.01$ & $0.74 \pm 0.00$ & $3.45 \pm 0.02$ & $25.76 \pm 0.32$ & $6.41 \pm 0.01$ \\
\hline Go-27 & $15.23 \pm 0.11$ & $8.84 \pm 0.28$ & $3.50 \pm 0.02$ & $8.65 \pm 0.17$ & $1.56 \pm 0.21$ & $\mathrm{ND}$ * & $3.11 \pm 0.13$ & $31.03 \pm 1.35$ & $5.49 \pm 0.06$ \\
\hline Go-28 & $16.78 \pm 0.20$ & $9.24 \pm 0.01$ & $4.84 \pm 0.02$ & $24.39 \pm 0.03$ & $0.56 \pm 0.00$ & $16.94 \pm 0.03$ & $6.72 \pm 0.03$ & $19.65 \pm 0.03$ & $5.89 \pm 0.01$ \\
\hline Go-29 & $13.48 \pm 0.01$ & $8.46 \pm 0.00$ & $3.33 \pm 0.00$ & $13.47 \pm 0.03$ & $0.66 \pm 0.01$ & $8.75 \pm 0.14$ & $4.38 \pm 0.02$ & $20.02 \pm 0.01$ & $6.44 \pm 0.03$ \\
\hline Go-30 & $13.08 \pm 0.03$ & $8.78 \pm 0.02$ & $4.11 \pm 1.32$ & $11.56 \pm 0.08$ & $1.06 \pm 0.01$ & $0.69 \pm 0.01$ & $3.72 \pm 0.06$ & $32.89 \pm 0.08$ & $7.31 \pm 0.12$ \\
\hline Go-31 & $14.41 \pm 0.30$ & $8.08 \pm 0.01$ & $2.82 \pm 0.01$ & $8.82 \pm 0.08$ & $2.93 \pm 0.01$ & $0.69 \pm 0.03$ & $3.05 \pm 0.02$ & $24.25 \pm 0.11$ & $6.15 \pm 0.02$ \\
\hline Go-32 & $13.04 \pm 0.84$ & $8.08 \pm 0.12$ & $2.93 \pm 0.06$ & $10.55 \pm 0.54$ & $0.44 \pm 0.02$ & $\mathrm{ND}^{*}$ & $2.61 \pm 0.25$ & $24.93 \pm 1.19$ & $6.66 \pm 0.13$ \\
\hline Go-33 & $10.67 \pm 0.23$ & $\mathrm{ND}^{*}$ & $\mathrm{ND}^{*}$ & $30.50 \pm 3.01$ & $1.31 \pm 0.13$ & $15.54 \pm 2.16$ & $20.23 \pm 2.21$ & $16.35 \pm 0.07$ & $5.39 \pm 0.03$ \\
\hline Go-34 & $25.71 \pm 0.50$ & $9.59 \pm 0.04$ & $3.31 \pm 0.01$ & $9.11 \pm 0.07$ & $0.53 \pm 0.01$ & $1.07 \pm 0.04$ & $2.24 \pm 0.01$ & $20.09 \pm 0.00$ & $5.99 \pm 0.01$ \\
\hline Go-35 & $21.43 \pm 0.06$ & $7.97 \pm 0.01$ & $3.98 \pm 0.07$ & $8.88 \pm 0.03$ & $0.49 \pm 0.01$ & $0.74 \pm 0.02$ & $2.22 \pm 0.02$ & $19.27 \pm 0.06$ & $5.73 \pm 0.01$ \\
\hline
\end{tabular}


Cadaverine and tryptamine are other frequently found BAs in foods; however, no legal limit has been established for cadaverine and tryptamine in foods. Cadaverine has no significant toxicity; however, its presence synergistically enhances the toxic effect of histamine. Cadaverine also has a negative impact on the catabolism of histamine by interfering with histamine detoxification [38-40]. Herein, the level of cadaverine ranged from $0.31-3.63 \mathrm{mg} / \mathrm{kg}$ in the gochujang samples, with the highest level found in Go-11 $(3.63 \pm 0.02 \mathrm{mg} / \mathrm{kg}$ ) (Figure 2 and Table 2). The results align well with those of Cho et al. [19] and Kim et al. [20], showing the similar presence of cadaverine in different gochujang samples. The involvement of tryptamine in increasing blood pressure, which can lead to hypertension has been well reported in the literature [41]. Therefore, its presence in food is critical to the establishment of food safety. Tryptamine was either not detected or detected up to $12.11 \mathrm{mg} / \mathrm{kg}$ in different gochujang samples, with the highest amount detected in Go-24 (12.11 $\pm 0.05 \mathrm{mg} / \mathrm{kg}$ ) (Figure 2 and Table 2). Among the 35 gochujang products, $8.57 \%$ of the products had $>10 \mathrm{mg} / \mathrm{kg}$ of tryptamine. Such findings aligned well with those of Lee et al. [21], who determined tryptamine levels in eight gochujang products. However, the amount of tryptamine $(0.1-4.1 \mathrm{mg} / \mathrm{kg})$ in the gochujang products tested in their study was lower than that detected in the products tested in the present study.

All analyzed gochujang products harbored low quantity of BAs. Major factors that affect the quantity of BAs in traditional gochujang products include manufacturing practice, ratio and quality of raw material, the microbial consortium involved during fermentation, fermentation environment, and the duration of the fermentation process. The predominant bacterial species found in gochujang are Bacillus subtilis, followed by Bacillus licheniformis and Bacillus amyloliquefaciens, which are well known producers of BAs due to the presence of decarboxylase enzyme $[42,43]$. The decarboxylase activities in these bacteria markedly vary between the strains and may be a major cause of the difference in the quantity of BAs in various gochujang products [10]. Besides bacteria, some studies reported that yeast can also produce BAs. Particularly, Zygosaccharomyces rouxii is predominant in traditional gochujang. However, yeasts have been considered to produce only negligible amounts of BAs $[13,44]$.

A considerably high number of BAs was found in Go-22, followed by Go-28, which contains soybean, cheonggukjang (a fast fermented soybean paste), and meju which provide a protein rich environment for the production of BAs. Go-8, Go-22, and Go-28 also had higher amounts of free amino nitrogen (data not shown), which may influence the formation of BAs in those products as they are the readily available substrates for the synthesis of various types of BAs. In general, red pepper is the major ingredient in traditional gochujang products [1]. Red pepper contains capsaicin as a bioactive compound, which has been evaluated for its inhibitory effect against the formation of BAs [7]; this might be one of the reasons for the low production of BA in gochujang products. Moreover, gochujang has an acidic $\mathrm{pH}$ and high salinity, which are known to affect the production of BA and control the growth of BA producing microbes [7]. Further, numerous strains of Bacillus sp. isolated from the different fermented foods are found to actively degrade BAs [13], which supports the low amount of BA in gochujang products owing to the predominance of these species in traditional gochujang products. The additives (for example, alcohol added in G-20 gochujang product) mixed with gochujang may also play a role in the reduction of BAs either by decreasing BA production or inhibiting the growth of BAs producing microbes [7].

The overall results indicated that the mean and median values of the estimated BAs, namely agmatine, tryptamine, 2-phenylethylamine, putrescine, cadaverine, histamine, tyramine, spermidine, and spermine in gochujang products from the traditional cottage industry were $14.60,8.24,3.96,13.88,0.98,2.72,6.47,23.95$, and $6.09 \mathrm{mg} / \mathrm{kg}$, and 13.48, 8.04, 3.03, $9.37,0.57,0.82,3.45,24.09$, and $6.00 \mathrm{mg} / \mathrm{kg}$, respectively (Figure 2). Similarly, the minimum and maximum amounts of putrescine, cadaverine, tyramine, spermidine, and spermine in the gochujang samples were 7.60, 0.27, 2.15, 14.96, and $4.68 \mathrm{mg} / \mathrm{kg}$, and 56.72, 3.63, $52.34,36.93$, and $7.31 \mathrm{mg} / \mathrm{kg}$, respectively. Agmatine, tryptamine, histamine, and 2phenylethylamine were either not detected or detected up to maximum amounts of 29.78, 
$12.11,16.94$, and $26.23 \mathrm{mg} / \mathrm{kg}$, respectively, in the gochujang samples. Although few samples had 100-175 mg/ $\mathrm{kg}$ of total BA, the average total BA content in the gochujang samples was $80.09 \mathrm{mg} / \mathrm{kg}$, which is quite lower than the toxic limit set by many government entities and independent researchers. The present results indicate that all tested gochujang products from the traditional cottage industry are safe for consumption in terms of BA content.

Multivariate analysis using PCA was performed to segregate the BAs in different gochujang samples (Figure 3). PC1 separated Go-1, Go-8, Go-11, Go-18, Go-22, Go-23, Go-28, and Go-29, (positive PC1 values) from the other samples (Figure 3). Further, PC2 explained the variance and separated Go-4, Go-6, Go-7, Go-9, Go-12, Go-13, Go-15, Go-16, Go-20, Go-21, Go-23, Go-25, Go-28, Go-29, and Go-35 from the other samples (Figure 3). PCA separated and derived the different clusters of 35 gochujang samples based on their BAs. The histamine content significantly influenced Go-29, Go-28, and Go-11 gochujang products, whereas the spermine, agmatine, cadaverine, 2-phenylethylamine, tyramine, and putrescine content influenced Go-1, Go-18, Go-23, Go-22, and Go-8. However, a few of the gochujang samples did not show any variation in BA quantities (Figure 3). The spermidine and tryptamine contents displayed significant variance with the other tested BAs, and maximum variance was found between histamine and spermidine, followed by tryptamine. To the best of our knowledge, no study has categorized the BAs in gochujang samples using multivariate PCA; however, a few studies have used PCA to represent the BA profiling in food products $[45,46]$.

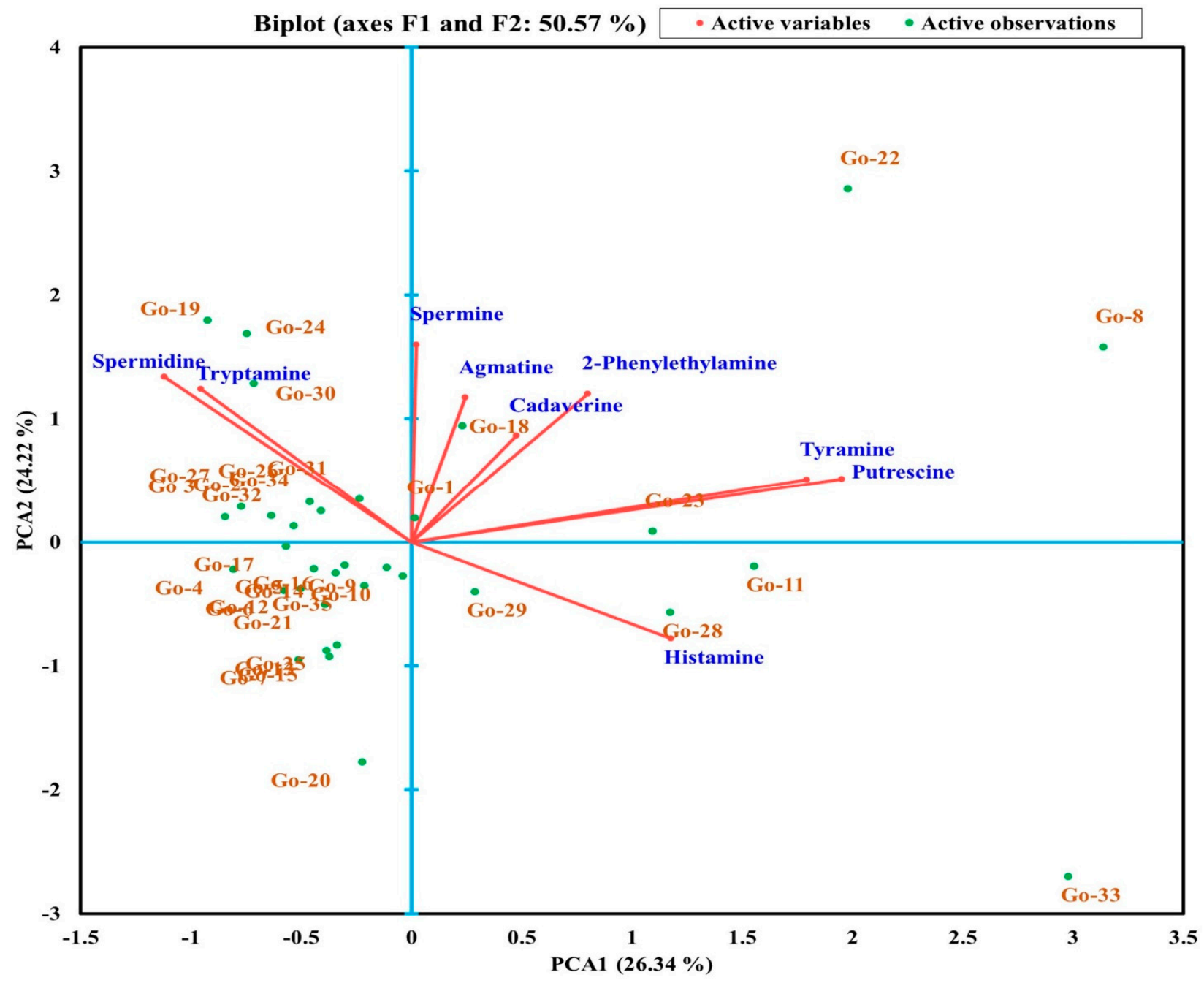

Figure 3. Principal component analysis of biogenic amines in different gochujang products from the traditional cottage industry. 


\section{Conclusions}

All the gochujang products from the cottage industry had markedly lower levels of BAs than the suggested toxicity limits, indicating good manufacturing practice, excellent hygienic conditions, and the selection of high-quality raw material for gochujang preparation. The major reason for low levels of BAs in the analyzed traditional gochujang might be the use of raw materials containing low proteinaceous substances, except meju (fermented soybeans). This study revealed the safety of tested traditional gochujang produced by the cottage industry in different provinces of Korea. The results also revealed that the levels of BAs in the cottage-based traditional gochujang products posed no issue of toxicity, thereby indicating that these products are safe for human consumption.

Author Contributions: Conceptualization, M.K.; methodology, S.R., A.B., S.L., A.-r.J., J.-S.L. and S.-Y.K.; software, S.R., A.B. and S.L.; validation, S.R., A.B., S.L., A.-r.J. and J.-S.L.; formal analysis, S.R., A.B., S.L., A.-r.J. and J.-S.L.; investigation, S.R., A.B., S.L., A.-r.J. and J.-S.L.; resources, S.-Y.K. and M.K.; data curation, S.R., A.B., S.L., A.-r.J. and J.-S.L.; writing—original draft preparation, S.R., A.B. and S.L.; supervision, M.K.; project administration, S.-Y.K. and M.K.; and funding acquisition, M.K. All authors have read and agreed to the published version of the manuscript.

Funding: This research was funded by the Cooperative Research Program for Agricultural Science and Technology Development, Rural Development Administration, Republic of Korea [Project No. PJ013833].

Institutional Review Board Statement: Not applicable.

Informed Consent Statement: Not applicable.

Data Availability Statement: Data is contained within the article.

Acknowledgments: The authors thank the Core Research Support Center for Natural Products and Medicinal Materials (CRCNM) for providing technical support related to sample preparation.

Conflicts of Interest: The authors declare no conflict of interest.

\section{References}

1. Information Statistics System, 2018, Food Market Newsletter-2018. Available online: https://www.atfis.or.kr/article/M0010100 00/view.do?articleId=3034 (accessed on 19 February 2021).

2. CODEX Alimentarius. Regional Standard for Gochujang (Asia): CODEX STAN 294R-2009. 2009. Available online: http:/ / www.fao.org/fao-who-codexalimentarius/sh-proxy/en/?lnk=1\&url=https\%253A\%252F\%252Fworkspace.fao.org\%25 2Fsites\%252Fcodex\%252FStandards\%252FCXS\%2B294R-2009\%252FCXS_294Re.pdf (accessed on 31 December 2018).

3. Kim, H.E.; Han, S.Y.; Kim, Y.S. Quality characteristics of Gochujang meju prepared with different fermentation tools and inoculation time of Aspergillus oryzae. Food Sci. Biotechnol. 2010, 19, 1579-1585. [CrossRef]

4. Shin, D.; Jeong, D. Korean traditional fermented soybean products: Jang. J. Ethn. Foods. 2015, 2, 2-7. [CrossRef]

5. Kim, D.H.; Choi, H.J. Physicochemical properties of kochujang prepared by Bacillus sp. koji. Korean J. Food Sci. Technol. 2003, 35, 1174-1181.

6. Burges, P. Modification of a traditional Korean food product (gochujang) to enhance its consumer acceptability as an ethnic food. J. Ethn. Foods. 2014, 1, 13-18. [CrossRef]

7. Naila, A.; Finlt, S.; Fletcher, G.; Bremer, P.; Meerdink, G. Control of biogenic amines in food-existing and emerging approaches. J. Food Sci. 2010, 75, 139-150. [CrossRef]

8. Askar, A.; Treptow, H. Biogene Amine in Lebensmitte. In Vorkommen, Bedeutung und Bes-Timmung, 1st ed.; Verlag Eugen Ulmer: Stuttgart, Germany, 1986; pp. 21-74.

9. Yoon, S.H.; Koh, E.; Choi, B.; Moon, B. Effects of soaking and fermentation time on biogenic amines content of Maesil (Prunus Mume) extract. Foods 2019, 8, 592. [CrossRef]

10. Martuscelli, M.; Esposito, L.; Mastrocola, D. Biogenic Amines' Content in Safe and Quality Food. Foods 2021, 10, 100. [CrossRef]

11. Park, Y.K.; Lee, J.H.; Mah, J.H. Occurrence and reduction of biogenic amines in kimchi and Korean fermented seafood products. Foods 2019, 8, 547. [CrossRef]

12. Del Rio, B.; Redruello, B.; Linares, D.M.; Ladero, V.; Fernandez, M.; Martin, M.C.; Ruas-Madiedo, P.; Alvarez, M.A. The dietary biogenic amines tyramine and histamine show synergistic toxicity towards intestinal cells in culture. Food Chem. 2017, 218, 249-255. [CrossRef]

13. Mah, J.-H.; Park, Y.K.; Jin, Y.H.; Lee, J.-H.; Hwang, H.-J. Bacterial Production and Control of Biogenic Amines in Asian Fermented Soybean Foods. Foods 2019, 8, 85. [CrossRef] 
14. Kim, M.K.; Mah, J.H.; Hwang, H.J. Biogenic amine formation and bacterial contribution in fish, squid and shellfish. Food Chem. 2009, 116, 87-95. [CrossRef]

15. Shin, D.H.; Kim, D.H.; Choi, U.; Lim, M.S.; An, E.Y. Changes in microflora and enzymes activities of traditional gochujang prepared with various raw materials. Korean J. Food Nutr. 1997, 29, 901-906.

16. Shin, D.; Ahn, E.; Kim, Y.; Oh, J. Changes in physicochemical characteristics of gochujang prepared with different koji during fermentation. Korean J. Food Sci. Technol. 2001, 33, 256-263.

17. Shin, D.; Ahn, E.; Kim, Y.; Oh, J. Changes in the microflora and enzyme activities of kochujang prepared with different koji during fermentation. Korean J. Food Sci. Technol. 2001, 33, 94-99.

18. Kim, D.H.; Kwon, Y.M. Effect of storage conditions on the microbiological and physicochemical characteristics of traditional Kochujang. Korean J. Food Sci. Technol. 2001, 33, 589-595.

19. Cho, T.Y.; Han, G.H.; Bahn, K.N.; Son, Y.W.; Jang, M.R.; Lee, C.H.; Kim, S.H.; Kim, D.B.; Kim, S.B. Evaluation of biogenic amines in Korean commercial fermented foods. Korean J. Food Sci. Technol. 2006, 38, 730-737.

20. Kim, T.K.; Lee, J.I.; Kim, J.H.; Mah, J.H.; Hwang, H.J.; Kim, Y.W. Comparison of ELISA and HPLC methods for the determination of biogenic amines in commercial doenjang and gochujang. Food Sci. Biotechnol. 2011, 20, 1747-1750. [CrossRef]

21. Lee, J.I.; Oh, Y.K.; Kim, J.H.; Kim, Y.W. Rapid enzymatic assay of biogenic amines in doenjang and gochujang using amine oxidase. Food Sci. Biotechnol. 2013, 22, 1131-1136. [CrossRef]

22. Ramalingam, S.; Dhatchanamoorthi, I.; Arumugam, A.; Bahuguna, A.; Krishnamoorthy, M.; Lee, J.S.; Devarajan, N.; Kim, M. Functional, nutritional, antinutritional, and microbial assessment of novel fermented sugar syrup fortified with pre-mature fruits of Totapuri mango and star gooseberry. LWT_Food Sci. Technol. 2021, 136, 110276. [CrossRef]

23. Santos, M.S. Biogenic amines: Their importance in foods. Int. J. Food Microbial. 1996, 29, 213-231. [CrossRef]

24. Food and Drug Administration (US FDA). Fish and Fishery Products Hazards and Controls Guidance, 4th ed.; Center for Food Safety and Applied Nutrition: Rockville, MD, USA, 2011.

25. European Commission Regulation (EC). No 2073/2005 Microbiological criteria for foodstuffs (Text with EEA relevance). Off. J. Eur. Union 2005, 338, 1-26.

26. Food and Agriculture Organization of the United Nations/World Health Organization (FAO/WHO). Joint FAO/WHO Expert Meeting on the Public Health Risk of Histamine and Other Biogenic Amines from Fish and Fishery Products; World Health Organization: Rome, Italy, 2013. Available online: https:/ / apps.who.int/iris/handle/10665/89216 (accessed on 5 April 2021).

27. The Ministry of Food and Drug Safety (MFDS). Food Code, Notification No. 2017-57; MFDS: Osong, Korea, 2017.

28. China National Standards (CNS). GB 2733-2015 National Food Safety Standards for Fresh and Frozen Animal Aquatic Products; Standards Press of China: Beijing, China, 2016.

29. Asatoor, A.M.; Levi, A.J.; Milne, M.D. Tranylcypromine and cheese. Lancet 1963, 2, 733. [CrossRef]

30. ten Brink, B.; Damink, C.; Joosten, H.M.L.J.; Huis in't Veld, J.H.J. Occurrence and formation of biologically active amines in foods. Int. J. Food Microbiol. 1990, 11, 73-84. [CrossRef]

31. Chou, H.T.; Kwon, D.H.; Hegazy, M.; Lu, C.D. Transcriptome analysis of agmatine and putrescine catabolism in Pseudomonas aeruginosa PAO1. J. Bacteriol. 2008, 190, 1966-1975. [CrossRef] [PubMed]

32. Arena, M.E.; Manca de Nadra, M.C. Biogenic amine production by Lactobacillus. J. Appl. Microbiol. 2001, 90, 158-162. [CrossRef] [PubMed]

33. Kalăc, P. Health effects and occurrence of dietary polyamines: A review for the period 2005-mid 2013. Food Chem. 2014, 161, 27-39. [CrossRef] [PubMed]

34. Kalăc, P.; Krausová, P. A review of dietary polyamines: Formation, implications for growth and health and occurrence in foods. Food Chem. 2005, 90, 219-230. [CrossRef]

35. Shalaby, A.R. Significance of biogenic amines to food safety and human health. Food Res. Int. 1996, 29, 675-690. [CrossRef]

36. Til, H.P.; Falke, H.E.; Prinsen, M.K.; Willems, M.I. Acute and subacute toxicity of tyramine, spermidine, spermine, putrescine and cadaverine in rats. Food Chem. Toxicol. 1997, 35, 337-348. [CrossRef]

37. Stratton, J.E.; Hutkins, R.W.; Taylor, S.L. Biogenic amines in cheese and other fermented foods: A review. J. Food Prot. 1991, 54, 460-470. [CrossRef]

38. Rice, S.L.; Eitenmiller, R.R.; Koehler, P.E. Biologically active amines in food: A review. J. Milk Food Technol. 1976, 39, 353-358. [CrossRef]

39. Taylor, S.L. Histamine Poisoning Associated with Fish, Cheese, and Other Foods; Monografia (VPH/FOS/85.1); World Health Organization: Geneva, Switzerland, 1985; p. 17.

40. Sattler, J.; Hafner, D.; Klotter, H.J.; Lorenz, W.; Wagner, P.K. Food induced histaminosis as an epidemiological problem: Plasma histamine elevation and haemodynamic alterations after oral histamine administration and blockade of diamine oxidase (DAO). Agents Actions 1988, 23, 361-365. [CrossRef]

41. Joosten, H.M.L.G. The biogenic amine contents of Dutch cheese and their toxicological significance. Neth. Milk Dairy J. 1988, $42,25-42$.

42. Ryu, J.-A.; Kim, E.; Kim, M.-J.; Lee, S.; Yoon, S.-R.; Ryu, J.-G.; Kim, H.-Y. Physicochemical Characteristics and Microbial Communities in Gochujang, a Traditional Korean Fermented Hot Pepper Paste. Front. Microbiol. 2021, 11, 620478. [CrossRef]

43. Baek, S.-Y.; Gil1, N.-Y.; Han, M.-H.; Kang, H.-Y.; Lee, H.-Y.; Yoon, Y.-S.; Lee, J.; Song, Y.-E.; Lee, S.-K.; Ryu, J.-A.; et al. Study on relationship between quality characteristics and exterior environment of the Korean traditional gochujang produced in 2018 by 8 regions of Korea. Korean J. Food Preserv. 2019, 26, 2287-7428. [CrossRef] 
44. Qi, W.; Hou, L.H.; Guo, H.L.; Wang, C.L.; Fan, Z.C.; Liu, J.F.; Cao, X.H. Effect of salt-tolerant yeast of Candida versatilis and Zygosaccharomyces rouxii on the production of biogenic amines during soy sauce fermentation. J. Sci. Food. Agric. 2014, 94, 1537-1542. [CrossRef] [PubMed]

45. Reis, G.C.; Custódio, F.B.; Botelho, B.G.; Guidi, L.R.; Gloria, M.B.A. Investigation of biologically active amines in some selected edible mushrooms. J. Food Compost. Anal. 2020, 86, 103375. [CrossRef]

46. Héberger, K.; Csomós, E.; Simon-Sarkadi, L. Principal component and linear discriminant analyses of free amino acids and biogenic amines in Hungarian wines. J. Agric. Food Chem. 2003, 51, 8055-8060. [CrossRef] [PubMed] 